This paper has been originally published at:

G. Haase, D. Martin, and G. Offner. Towards RBF Interpolation on Heterogeneous HPC Systems. In I. Lirkov, S. Margenov, J. Wasniewski (eds.) Large Scale Scientific Computing LSSC'15, 2015, Sozopol, Bulgaria, LNCS, volume 9374, Springer, Heidelberg, p. 182-190. doi : 10.1007/978-3-319-26520-9_19. 


\title{
Towards RBF Interpolation on Heterogeneous HPC Systems
}

\author{
Gundolf Haase $^{1}$, Dirk Martin ${ }^{2,3}$, and Günter Offner ${ }^{3}$ \\ 1 Institute for Mathematics and Scientific Computing, University of Graz, Austria \\ gundolf.haase@uni-graz.at, \\ WWW home page: http://www. uni-graz.at/ ghaase \\ 2 VRVis Research Center, Vienna, Austria \\ 3 AVL List GmbH, Graz, Austria
}

\begin{abstract}
We present a general approach for the parallelization of the interpolation with radial basis functions $(\mathrm{RBF})$ on distributed memory systems, which might use various shared memory hardware as accelerator for the local subtasks involved. The calculation of an interpoland in general requires a global dense system to be solved. Iterative methods need appropriate preconditioning to achieve reasonable iteration counts. For the shared memory approach we use a special Krylov subspace method, namely the FGP algorithm. Addressing the distributed task we start with a simple block-Jacobi iteration with each block solved in parallel. Adding a coarse representation leads to a two-level block-Jacobi iteration with much better iteration counts and a wider applicability.
\end{abstract}

\section{Introduction}

The numerical treatment of many simulation problems in science and industry has to handle changing computational domains originating from the given PDE (system) or from design variables in optimization and optimal control problems. A re-meshing in case of a direct problem, i.e. the PDE, is possible but rather inefficient because all mesh-dependent data have to be reallocated and recomputed. In the context of an optimization problem the re-meshing would destroy the continuous differentiability of the objective functional and therefore we are forced to apply a mesh deformation instead.

We use interpolation with radial basis functions ( $\mathrm{RBF}$ interpolation) for mesh deformation as proposed in [4]. In this paper we focus on the parallelization of RBF interpolation with its application for mesh deformation in view. Within this context parallelization covers shared memory and distributed memory parallel computing.

Calculating an RBF interpoland requires the solution of a dense system of linear equations. There have been several achievements to overcome the illconditioning of the linear system $[2,3,16]$. Nevertheless a direct solution of the system is inhibited, if the problem size exceeds certain limits, thus iterative methods have to be used. Due to the ill-conditioning of the linear system, some preconditioning has to be applied. One way is to use domain decomposition 
methods $[2,13,19]$. We employ a Krylov-subspace method that uses approximate Lagrange functions as preconditioner, namely the FGP algorithm $[9,10]$ for a shared memory solution. Our reasonable approach to a distributed memory solution is applying well known domain decomposition methods.

Due to our application of deforming given computational meshes our data distribution is predetermined. In particular we work on distributed finite volume discretizations with one cell-layer overlap.

The remaining paper is organized as follows. Section 2 gives a short introduction to RBF interpolation. We feature the FGP algorithm and our approach to an efficient shared memory implementation in section 3. Section 4 covers the distributed memory approach. We present some numerical results in section 5 and annotate some conclusions in section 6 .

\section{Interpolation with Radial Basis Functions}

This introduction to RBF interpolation closely follows [6]. Further analysis and treatment of basis functions with compact support can be found in [18].

Given is a set of points $\mathcal{X}=\left\{x_{i}\right\}_{i=1}^{N}$ in a domain $\Omega \subseteq \mathbb{R}^{d}$. A set of associated real function values $f_{i}=f\left(x_{i}\right)$ is assigned to these points. The function $f$ is usually unknown, but its existence is postulated for the reasonableness of the interpolation task. Sought is an approximating function $s: \Omega \rightarrow \mathbb{R}$ by interpolation. Restricted on the set $\mathcal{X}$ we request the interpolation condition

$$
\left.s\right|_{\mathcal{X}}=\left.f\right|_{\mathcal{X}}
$$

In the context of RBF interpolation we seek for an interpoland of the form

$$
s(x)=\sum_{i=1}^{N} \lambda_{i} \phi\left(\left\|x-x_{i}\right\|\right)+p(x), \quad \lambda_{i} \in \mathbb{R}, p \in \mathbb{P}^{M} .
$$

The polynomial term $p$ is required for the existence and uniqueness of a solution. The required degree $M$ for the existence of a solution depends in the choice of the basis function $\phi$, see [6]. We choose $\phi=\sqrt{r^{2}+c^{2}}, c \in \mathbb{R}$ and $M=0$ for our numerical examples in section 5 .

If the basis function requires a polynomial term $(M \geq 0)$ then the given set of points $\mathcal{X}$ has to be unisolvent w.r.t. polynomials of degree $M$, i.e., $\left.p\right|_{\mathcal{X}}=0 \Rightarrow$ $p \equiv 0$ for polynomials $p \in \mathbb{P}^{M}$.

Requiring the interpolation condition (1) in all given points and demanding a side condition on the coefficients of the polynomial term leads to a system of linear equations for the determination of the coefficients $\boldsymbol{\lambda}$ and $\boldsymbol{\pi}$ :

$$
\begin{array}{rlrl}
\sum_{i=1}^{N} \lambda_{i} \phi\left(\left\|x_{i}-x_{k}\right\|\right)+\sum_{j=1}^{M} \pi_{j} p_{j}\left(x_{k}\right) & =f\left(x_{k}\right), & & 1 \leq k \leq N, \\
\sum_{i=1}^{N} \lambda_{i} p_{l}\left(x_{i}\right) & =0, & 1 \leq l \leq M,
\end{array}
$$


or, in short notation

$$
\left(\begin{array}{cc}
\Phi & \Pi \\
\Pi^{\top} & 0
\end{array}\right)\left(\begin{array}{l}
\boldsymbol{\lambda} \\
\boldsymbol{\pi}
\end{array}\right)=\left(\begin{array}{l}
f \\
\mathbf{0}
\end{array}\right)
$$

\section{Accelerated Computation of Interpolands}

Analysis of the properties of the linear equations system (4) yields that a direct solution is inhibited, if the number of interpolation points exceeds certain limits. Therefore, we employ an iterative method $[9,10]$ to solve $(4)$.

The FGP algorithm is a Krylov-subspace method. The implemented version of the algorithm can be applied to interpolations with basis functions where constant polynomial terms are sufficient $(M=0)$.

Let $X$ denote the functional space spanned by functions of the form (2). The basis for the algorithm is the semi-inner product

$$
\langle s, t\rangle_{\phi}=-\boldsymbol{\lambda}^{\top} \Phi \boldsymbol{\mu} \quad \text { for } s, t \in X
$$

with $s(x)=\sum_{i=1}^{N} \lambda_{i} \phi\left(\left\|x-x_{i}\right\|\right)+\alpha$ for $\lambda_{i}, \alpha \in \mathbb{R}$ and $t(x)=\sum_{i=1}^{N} \mu_{i} \phi\left(\left\|x-x_{i}\right\|\right)+$ $\beta$ for $\mu_{i}, \beta \in \mathbb{R}$, induced by the radial basis function $\phi$. $\Phi$ denotes the associated kernel to $\phi$. Other basis functions may require a different sign at the definition of the semi-inner product. The semi-inner product $\langle\cdot, \cdot\rangle_{\phi}$ induces a semi-norm

$$
|s|_{\phi}=\langle s, s\rangle^{1 / 2}=\left(-\boldsymbol{\lambda}^{\top} \Phi \boldsymbol{\lambda}\right)^{1 / 2} .
$$

The FGP algorithm uses a linear operator $A: X \rightarrow X . A$ is chosen such, that for all iterations $k$ the resulting searching direction lies in the subspace of $X$ that is spanned by the functions $A^{l} s^{\star}, l=1, \ldots, k$, where $s^{\star}$ denotes the sought interpoland. In addition to this $A$ performs some preconditioning. $A$ is an approximation of the optimal preconditioning operator $A^{\mathrm{opt}}$. The operator $A^{\text {opt }}$ can be derived from the interpolation tasks

$$
\hat{u}_{j}(x)=\sum_{i=1}^{N} \zeta_{j, i} \phi\left(x-x_{i}\right)+\beta, \quad \text { for } x \in \Omega, j=1, \ldots, N,
$$

due to the Lagrange conditions $\hat{u}_{j}\left(x_{i}\right)=\delta_{i j}, \quad i, j=1, \ldots, N$, where $\delta_{i j}$ denotes the Kronecker-delta.

For the construction of $A$ the functions $\hat{u}_{j}, j=1, \ldots, N$ are determined by solving the interpolation tasks on subsets of $\mathcal{X}$, that contain not more than $q$ interpolation centers. Generally the relation $q \ll N$ holds. These subsets are called $\mathcal{L}$-sets.

The main computational costs of an implementation lie in the construction of the $\mathcal{L}$-sets and the calculation of matrix-vector products with dense matrices. The authors in [11] show an efficient implementation including a modified setup stage and an approximated matrix-vector product by using a fast multipole method. We use an octree as spatial hierarchy to efficiently construct the $\mathcal{L}$ sets during the setup phase. We also use the same octree structure to employ a 
multipole-method [1]. The far field series expansion for the multiquadric basis function is described in [7].

Our approach to a shared memory parallelization of the matrix-vector product on CPUs using the OpenMP API [15] applies task based parallelization on octree box level.

We use a slightly different parallelization scheme for acceleration of the matrix-vector product on graphics processors using the CUDA programming model [8]. For the direct calculation of the matrix-vector multiplication the boundary-condition nodes (bc nodes) are organized in blocks. A block of bc nodes corresponds to a thread-block on the GPU. Within each block the result for each bc node is calculated by a single thread. Since the hierarchical structure that is used for the multipole approximation is an octree, the far field series expansion of each box in the 'evaluation region' (see [1]) of an octree box has to be evaluated for each node within this box. The thread-blocks on the GPU correspond to the octree boxes. Each thread on the GPU evaluates the polynomial for one node within the box. All threads read the (common) series coefficients to shared memory. If an octree box holds more nodes than the number of threads that are started within a block the box is virtually split in boxes that do not hold more nodes than threads-per-block each. After the completion of our work we learned about earlier results in [12].

\section{Parallel Computation of Interpolands}

The referred domain decomposition methods for the computation of RBF interpolands $[2,13,19]$ can be seen as preconditioning methods according to the classification in [17]. Thereby the solution of a large system over the domain $\Omega$ is subdivided into $P$ smaller problems over the subdomains $\Omega_{s}, s=1, \ldots, P$. The solutions of the smaller problems are used to construct a preconditioner for the solution of the large system.

Our general approach is to use the subdivision predetermined by the application and to apply the methods from section 3 to solve the regarding subproblems. Our given subdivision of the domain $\Omega$ consists of overlapping subdomains $\Omega_{s}$, i.e., $\Omega=\bigcup_{s=1}^{P} \Omega_{s}$ with $\Omega_{i} \cap \Omega_{j} \neq \emptyset, i \neq j$. Further let $\tilde{\Omega}_{s}$ denote the appropriate non-overlapping subdomains such that $\Omega=\bigcup_{s=1}^{P} \tilde{\Omega}_{s}$ holds with $\Omega_{i} \cap \Omega_{j}=\emptyset$, $i \neq j$.

Let $R_{s}$ denote the restriction matrix projecting a vector $\boldsymbol{x}$ from domain $\Omega$ onto a vector $\boldsymbol{x}_{s}=R_{s} \boldsymbol{x}$ on subdomain $\Omega_{s}$. Similarly $\tilde{R}_{s}$ denotes the restriction matrix which restricts a vector $\boldsymbol{x}$ from domain $\Omega$ onto a vector $\boldsymbol{x}_{s}$ on subdomain $\tilde{\Omega}_{s}$. For the non-overlapping subdivision a vector over the domain $\Omega$ can be composed by applying the transposed mapping operations $\tilde{R}^{\top}$ on the local vectors $\boldsymbol{x}_{s}$

$$
\boldsymbol{x}=\sum_{s=1}^{P} \tilde{R}^{\top} \boldsymbol{x}_{s}
$$




\subsection{Block-Jacobi Iteration}

The authors in [19] show the utilization of a Restricted Additive Schwarz method (RASM) as preconditioner for the calculation of an RBF interpoland. The proposed method is applicable for positive (negative) definite basis functions, thus no polynomial term is required in (2).

The $n$-th iteration of the RASM to calculate the solution of (4) is

$$
\boldsymbol{\lambda}^{(n+1)}=\boldsymbol{\lambda}^{(n)}+\sum_{i=1}^{P} \tilde{R}_{i}^{\top} \Phi_{i}^{-1} R_{i}\left(\boldsymbol{f}-\Phi \boldsymbol{\lambda}^{(n)}\right),
$$

where $\Phi_{i}$ denotes the system matrix of the subproblem restricted to the domain $\Omega_{i}$. This can be extended to conditionally positive (negative) basis functions. Let $\Pi_{i}$ denote the respective matrix blocks of the restricted system.

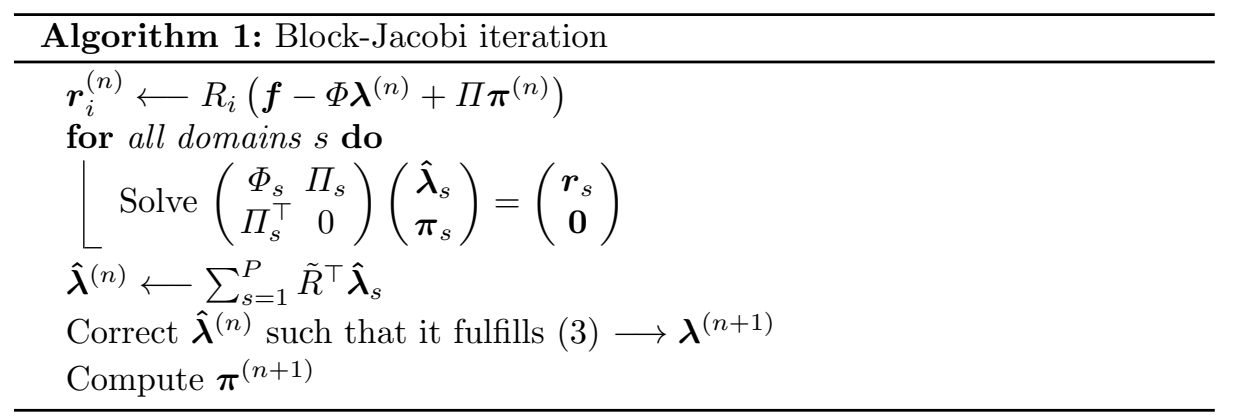

Depending on the size of the subproblem we either use a direct method or employ the FGP algorithm to find a solution for the local subproblems. The authors in [13] show that it is sufficient to calculate an approximative solution of the local subproblem requiring an error relative to the current residual in each iteration.

\subsection{Two-Level Block-Jacobi Iteration}

The authors in [2] sketched a simplified two-level domain decomposition method for RBF interpolation fitting. The two-level algorithm represented on page 6 requires a coarse grid representation $\mathcal{Y}$ consisting of interpolation centers from each subdomain $\tilde{\Omega}_{s}$. Let $R$ y denote the restriction matrix that restricts a global vector to the coarse grid interpolation centers and indices $y$ denote restricted vectors and matrices accordingly. We again use either a direct method or the FGP algorithm to solve the subproblems.

\section{$5 \quad$ Numerical Examples}

In this section we present timing results for performance tests of the method described in section 3 and iteration counts for the parallel computation of interpolands described in section 4 . 


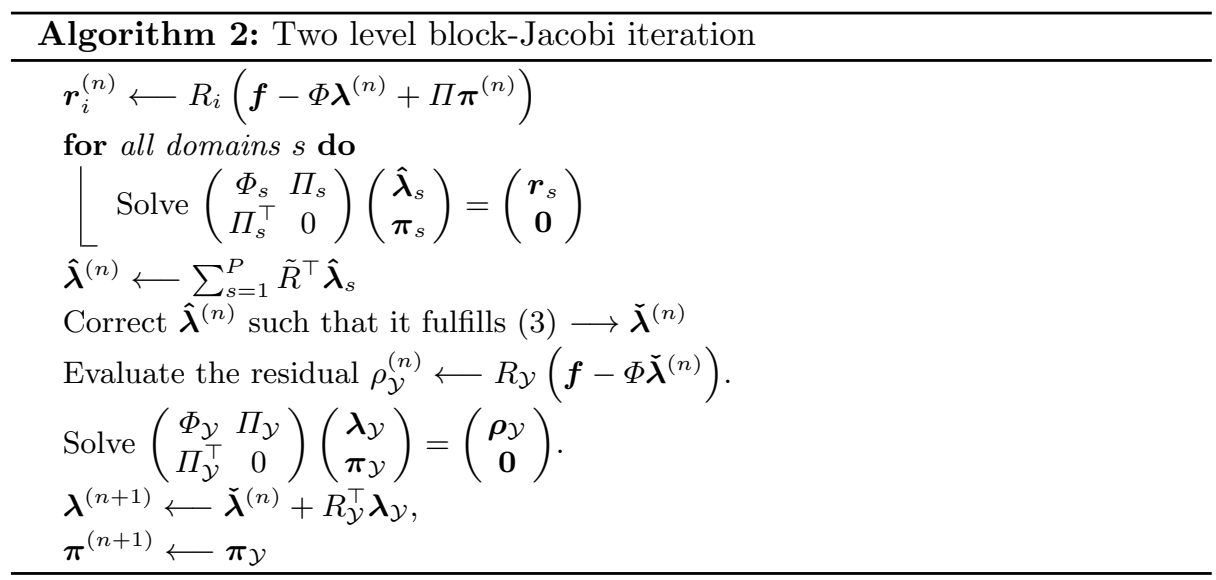

The test system is equipped with two Intel Xeon E5-2450 processors with 8 cores each. The OpenMP parallelized $\mathrm{C}++$ code uses 16 threads. The used GPGPU accelerator is an Nvidia Tesla K20Xm. Table 1 lists timings for the brute force computation and an approximation using a multipole method of a single matrix-vector product. The GPU version is faster by one order of magnitude

\begin{tabular}{|c|c|c|c|c|c|c|}
\hline \multirow{2}{*}{$\begin{array}{r}\# \text { bc } \\
\text { nodes }\end{array}$} & \multicolumn{2}{|c|}{ brute force } & \multicolumn{4}{|c|}{ multipole method } \\
\hline & $\mathrm{CPU}[\mathrm{s}]$ & GPU [s] & $\mathrm{CP}$ & CPU eva & GPU setu & GPI \\
\hline & $1.09 \mathrm{E}-02$ & $5.61 \mathrm{E}-04$ & $2.35 \mathrm{E}-02$ & $2.67 \mathrm{E}-02$ & $1.15 \mathrm{E}-02$ & $2.97 \mathrm{E}-03$ \\
\hline 156 & 9. & 3 & 2.0 & 5.1 & 1 & 3. \\
\hline 3542 & 1.06 & 2.4 & 2.4 & 1.4 & 02 & 03 \\
\hline 990 & 7.03E-02 & $6.32 \mathrm{E}-03$ & 2.30E-02 & 3.4 & E-02 & $4.49 \mathrm{E}-03$ \\
\hline 98 & $5.59 \mathrm{E}-01$ & 7.40E-02 & $8.00 \mathrm{E}-02$ & $5.54 \mathrm{E}-01$ & $1.65 \mathrm{E}-01$ & 2.39E-02 \\
\hline & $2.84 \mathrm{E}+00$ & $.91 \mathrm{E}-01$ & $1.71 \mathrm{E}-01$ & $2.15 \mathrm{E}+00$ & $3.44 \mathrm{E}-01$ & $4.77 \mathrm{E}-02$ \\
\hline
\end{tabular}

Table 1. Timings for a single matrix-vector product in seconds.

at least for both methods and the multipole method becomes superior with increasing problem size. Observing the multipole evaluation on the GPU for problem sizes below 10.000 nodes reveals the kernel invocation overhead. In contrast to the CPU implementation the octree boxes in the multipole method have to padded with additional zeros in order to achieve coalesce memory access on the GPU. The fill rate of these octree boxes changes with the tree depth and influences directly the run time such that the observed speed-up varies. This can be seen in Tab. 1 for the multipole evaluation wherein the GPU timings follow closely the expected run time $\mathcal{O}(N \log N)$ but the $\mathrm{CPU}$ performs even better, i.e., on 39802 nodes. The double precision peak performance and memory bandwidth of the Tesla K20Xm is 5 times better than those of the two Xeon processors. This 
factor is observed for the brute force approach while the multipole evaluation on GPU is considerably better vectorized than on CPU. A detailed discussion of the results can be found in [14].

Table 2 compares the iteration counts for the block-Jacobi and the two-level block-Jacobi preconditioning described in section 4. We imposed test function F3 as in [5] as boundary conditions on $N$ nodes distributed over a sphere. We test the effect of a fixed-size coarse grid $(|\mathcal{Y}|=200)$ versus an adjusted coarse grid size $(|\mathcal{Y}| \approx N / 8)$, arising as the natural choice for a prospective multi-level preconditioning based on an octree hierarchy. The iteration count indicates clearly that

\begin{tabular}{r|ccc|cccc|cccc} 
& \multicolumn{3}{|c|}{ Block-Jacobi } & \multicolumn{4}{c|}{ Two-level block-Jacobi } \\
& \multicolumn{3}{|c|}{} & \multicolumn{4}{|c|}{$|\mathcal{Y}|=200$} & \multicolumn{4}{c}{$|\mathcal{Y}| \approx N / 8$} \\
\hline & 2 & 4 & 8 & 2 & 4 & 8 & 16 & 2 & 4 & 8 & 16 \\
\hline 416 & 12 & 23 & 39 & 3 & 4 & 4 & 5 & 5 & 6 & 8 & 9 \\
1832 & 36 & 178 & - & 5 & 6 & 7 & 8 & 5 & 6 & 7 & 8 \\
7472 & - & - & - & 9 & 11 & 13 & 13 & 5 & 6 & 7 & 7 \\
30080 & - & - & - & 30 & 29 & 40 & 39 & 5 & 5 & 6 & 6
\end{tabular}

Table 2. Iteration count for the parallel test case.

the two-level method is superior even though the parallelization of the coarse grid is still future work. We expect that the coarse grid parallelization combined with a further recursive coarsening will result in the fastest preconditioner.

\section{Conclusion}

We showed that an implementation of the FGP algorithm can be adapted to exploit the massive parallelism of GPGPU accelerator cards. The FGP algorithm evinces to be applicable as solution method for the arising sub-problems when the original problem is distributed. Extending the resulting block-Jacobi preconditioning with an additional coarse block results in a two-grid method with constant iteration count. The ideal coarsening factor $N / 8$ indicates the reasonableness of a further multilevel preconditioning for the RBF-interpolation based on the (already existing) octree hierarchy in $\mathbb{R}^{3}$.

This first application with the preconditioner in a simple iteration will be extended to a Krylov subspace method as outer iteration in future. Therein our preconditioner has to be adapted in order to fulfill the requirements of the respective method.

Further research is to utilize a wider set of programming standards, such as OpenACC and OpenMP 4.0, for many-core programming, which hold out the prospect of an incomplex transition to different accelerator hardware (e.g. Intel(CXeon $\mathrm{Phi}^{\mathrm{TM}}$ ). 


\section{References}

1. Beatson, R.K., Greengard, L.: A short course on fast multipole methods. Wavelets, Multilevel Methods and Elliptic PDEs, pp. 1-37, Oxford University Press (1997).

2. Beatson, R.K., Light, W., Billings, S.: Fast Solution of the Radial Basis Function Interpolation Equations: Domain Decomposition Methods SIAM J. Sci. Comput., vol. 22, 5, pp. 1717-1740 (2001).

3. Beatson, R., Levesley, J., Mouat, C.: Better bases for radial basis function interpolation problems Comput. Appl. Math., vol. 236, pp. $434-446$ (2011).

4. de Boer, A., van der Schoot, M.S., Bijl, H.: Mesh Deformation Based on Radial Basis Function Interpolation. Comput. Struct., vol. 85, 11-14, pp. 784-795 (2007).

5. Bozzini, M.T., Rossini, M.F.: Multivariate approximation and interpolation with applications. Testing methods for 3D scattered data interpolation (Almunecar, 2001). Acad. Cienc. Exact.Fs.Qum. Nat.., pp. 111-135, (2002).

6. Buhmann, M.: Radial Basis Functions. Theory and Implementations. Cambridge monographs on applied and computational mathematics, Cambridge University Press, New York (2003).

7. Cherrie, J.B., Beatson, R.K., Newsam, G.N.: Fast Evaluation of Radial Basis Functions: Methods for Generalized Multiquadrics in Rn SIAM J. Sci. Comput., vol. 23, 5, pp. 1549-1571 (2001).

8. NVIDIA Corporation. CUDA programming guide 6.5 (2014). http://docs.nvidia.com/cuda/cuda-c-programming-guide/index.html.

9. Faul, A.C., Powell, M.J.D.: Krylov Subspace Methods for Radial Basis Function Interpolation. University of Cambridge, DAMP (1999).

10. Faul, A.C., Goodsell, G., Powell, M.J.D.: A Krylov subspace algorithm for multiquadric interpolation in many dimensions. IMA J. Numer. Anal., vol. 25, 1, pp. 1-24 (2005).

11. Gumerov, N. ,Duraiswami, R.: Fast Radial Basis Function Interpolation via Preconditioned Krylov Iteration. SIAM J. Sci. Comput., vol. 29, 5, pp. 1876-1899 (2007).

12. Gumerov, N., Duraiswami, R.: Fast multipole methods on graphics processors. $J$. Comput. Phys., vol. 227, pp 8290 - 8313, (2008).

13. Ling L., Kansa E.J.: Preconditioning for radial basis functions with domain decomposition methods. Math. Comput. Model., vol 40, 13, pp. 1413-1427 (2004).

14. Martin, D., Haase, G.: Interpolation with radial basis functions on GPGPUs using CUDA. Technical Report SFB-Report 2014-04, SFB MOBIS, University of Graz, (2014).

15. OpenMP Architecture Review Board. OpenMP Application Program Interface, Version 3.1 (2011). http://www.openmp.org/mp-documents/OpenMP3.1.pdf

16. Powell, M.J.D.: Some algorithms for thin plate spline interpolation to functions of two variables. Adv. Comput. Math., pp. 303-319 (1993).

17. Smith, B.F., Bjørstad, P.E., Gropp, W.D.: Domain decomposition: parallel multilevel methods for elliptic partial differential equations. Cambridge University Press, New York (1996).

18. Wendland, H.: Scatterred Data Approximation. Cambridge monographs on applied and computational mathematics, Cambridge University Press, New York (2010).

19. Yokota, R., Barba, L.A., Knepley, M.G. PetRBF A parallel O(N) algorithm for radial basis function interpolation with Gaussians Comput. Method. Appl. M., vol. 199, 25-28, pp. 1793-1804 (2010). 